Fakten und Statistiken 2006

\title{
6. Welthandel
}

Nadine Keim

\section{OpenEdition}

\section{Journals}

Electronic version

URL: http://journals.openedition.org/sjep/142

DOI: $10.4000 /$ sjep.142

ISSN: 1663-9677

\section{Publisher}

Institut de hautes études internationales et du développement

\section{Printed version}

Date of publication: 1 avril 2006

Number of pages: $81-101$

ISBN: 2-88247-062-2

ISSN: $1660-5926$

Electronic reference

Nadine Keim, «6. Welthandel », Schweizerisches Jahrbuch für Entwicklungspolitik [Online], 25-1 | 2006, Online erschienen am: 15 April 2010, abgerufen am 07 September 2020. URL : http:// journals.openedition.org/sjep/142; DOI : https://doi.org/10.4000/sjep.142 


\section{Welthandel*}

D ER WARENHANDEL nahm im Jahr 2004 volumenmässig um 9 Prozent bzw. wertmässig um 21 Prozent zu und verzeichnete mit diesen spektakulären Zahlen des kräftigste Wachstum seit 1979. Nordamerika und die Europäische Union haben ihre dominierende Position gefestigt - sie tragen an die zwei Drittel zum Welthandel mit Fertigwaren bei. Hingegen verliert die Schweiz nach und nach ihren Platz unter den wichtigsten Akteuren des Welthandels. Zu den prägenden Entwicklungen des Jahres gehörte auch das ausserordentliche Wirtschaftswachstum Chinas und Indiens und ihre zunehmende Rolle im Welthandel.

Für den Textil- und Bekleidungssektor bildete das Jahr 2005 mit dem Auslaufen des Multifaserabkommens und der Aufhebung der Einfuhrquoten den Auftakt zu einer neuen Etappe im Liberalisierungsprozess. Daraus ergibt sich ein Handelskrieg zwischen China, Indien und Pakistan einerseits und armen Ländern wie Bangladesch, Kambodscha sowie den Vereinigten Staaten und der Europäischen Union andererseits.

Der neue Generaldirektor der Welthandelsorganisation (WTO), Pascal Lamy, der am 1. September 2005 sein Amt übernahm, bemühte sich, die seit der Einigung vom Juli 2004 festgefahrenen Verhandlungen wieder in Schwung zu bringen. Die Ambitionen der Ministerkonferenz von Hongkong, die vom 13. bis zum 18. Dezember tagte, mussten jedoch heruntergeschraubt werden. Die einzigen konkreten Ergebnisse bilden die Annahme eines „Entwicklungspakets“ für die am wenigsten entwickelten Länder und die Festlegung eines Endtermins für die Aufhebung der Ausfuhrbeihilfen. Als Stolperstein in den Verhandlungen erwies sich insbesondere die Auslegung des Begriffs „Entwicklungsrunde“. Einige Länder, auch die Schweiz, verstehen darunter vor allem die Fortsetzung der Marktliberalisierung, andere hingegen die Gewährung eines echten wirtschaftspolitischen Handlungsspielraums ( „Policy Space“").

2005 feierte die WTO ihr zehnjähriges Bestehen. Die verschiedenen Veranstaltungen zum Jubiläum verdeutlichten, dass der Stillstand, der die WTO lähmt, auch auf bestimmte grundlegendere Umwälzungen zurückgeht: neue geopolitische Ordnung mit dem Erstarken der Entwicklungsländer, zunehmend komplexe Bündnisverhältnisse über die Nord-Süd-Kluft hinaus, Verkrustung der Institutionen. Erwähnenswert ist ferner, dass die Zivilgesellschaft im Jahr 2005 auf dem Gebiet des internationalen Handels eine sehr aktive Rolle spielte.

\subsection{Entwicklung der Handelsbeziehungen}

Das Jahr 2004 war vom spektakulären Wachstum des Handels vor dem Hintergrund eines kräftigen Produktionswachstums geprägt ${ }^{2}$. Die Warenausfuhren leg-

* Von Nadine Keim, lic. rer. pol., unabhängige Konsulentin.

Zur Definition des Konzepts „Policy Space“ siehe den diesbezüglichen Rahmentext unter Punkt 6.3.1.

2 WTO, International Trade Statistics 2005, Genf, 2005. Siehe auch WTO, World Trade Report 2005, Genf, 2005. 
ten volumenmässig um 9 Prozent zu und verzeichneten damit das beste Ergebnis seit dem Jahr 2000. Diese Beschleunigung ist weitgehend auf die ausgezeichneten Ergebnisse des Handels mit Fertigwaren zurückzuführen $(+10 \%)$, der doppelt so schnell zunahm wie 2003. Damit ist der Handel stärker gewachsen als die Produktion.

Tabelle 13: Weltausfuhren von Waren und kommerziellen Dienstleistungen, 2002-2004 (in Milliarden Dollar)

\begin{tabular}{llll}
\hline & $\mathbf{2 0 0 4}$ & $\mathbf{2 0 0 2}$ & $\mathbf{2 0 0 3}$ \\
\hline Waren & 8907 & 6270 & 7274 \\
\hline Kommerzielle Dienstleistungen & 2125 & 1570 & 1763 \\
\hline
\end{tabular}

Quelle:WT0, International Trade Statistics 2005, Genf, 2005, Tabelle 1, S. 3.

Der internationale Warenhandel verzeichnete 2004 (wertmässig, in Dollar) eine Zunahme von 21 Prozent, das kräftigste wertmässige jährliche Wachstum seit 1979, und erreichte 8907 Milliarden Dollar. Diese Entwicklung ist im Wesentlichen auf die starke Zunahme der Preise - gepaart mit einem weiteren Wertverlust $(9 \%)$ des amerikanischen Dollars gegenüber den Währungen der wichtigen europäischen Handelsnationen - und besonders der Erdölpreise zurückzuführen, die das höchste Niveau seit zwanzig Jahren erreichten. Der Wert der Brennstoffausfuhren und weiterer Erzeugnisse der Mineralindustrien stieg 2004 von 30 auf 45 Prozent und verzeichnete wie im Vorjahr eine deutlich raschere Zunahme als der Handel mit Fertigwaren (+20\%) oder mit landwirtschaftlichen Erzeugnissen $(+15 \%)$. Der Handel mit kommerziellen Dienstleistungen wuchs nominal um 18 Prozent und pendelte sich 2004 bei 2125 Milliarden Dollar ein.

Tabelle 14: Wertmässiges Wachstum des internationalen Warenhandels nach Regionen, 2004 (in Milliarden Dollar und Prozent)

\begin{tabular}{|c|c|c|c|c|c|c|c|c|}
\hline \multirow{3}{*}{ Region } & \multicolumn{4}{|c|}{ Exporte } & \multicolumn{4}{|c|}{ Importe } \\
\hline & \multirow{2}{*}{$\begin{array}{l}\text { Wert } \\
2004\end{array}$} & \multicolumn{3}{|c|}{ Jährliche Änderung (\%) } & \multirow{2}{*}{$\begin{array}{l}\text { Wert } \\
2004\end{array}$} & \multicolumn{3}{|c|}{ Jährliche Änderung (\%) } \\
\hline & & $2000-04$ & 2003 & 2004 & & $2000-04$ & 2003 & 2004 \\
\hline Ganze Welt & 8907 & 9 & 17 & 21 & 9250 & 9 & 17 & 21 \\
\hline Nordamerika & 1324 & 2 & 5 & 14 & 2013 & 5 & 8 & 17 \\
\hline Latein- und Zentralamerika & 276 & 9 & 13 & 30 & 237 & 4 & 5 & 27 \\
\hline Europa & 4031 & 11 & 19 & 19 & 4140 & 11 & 20 & 20 \\
\hline Europäische Union (25) & 3714 & 11 & 19 & 19 & 3791 & 10 & 20 & 19 \\
\hline $\begin{array}{l}\text { Gemeinschaft } \\
\text { Unabhängiger Staaten }\end{array}$ & 266 & 16 & 27 & 37 & 172 & 21 & 27 & 30 \\
\hline Russland & 183 & 15 & 27 & 35 & 96 & 21 & 25 & 27 \\
\hline Afrika & 232 & 12 & 25 & 32 & 212 & 13 & 22 & 27 \\
\hline Mittlerer Osten & 390 & 10 & 20 & 29 & 252 & 12 & 13 & 27 \\
\hline Asien & 2388 & 10 & 18 & 25 & 2224 & 10 & 19 & 27 \\
\hline China & 593 & 24 & 35 & 35 & 561 & 26 & 40 & 36 \\
\hline Japan & 566 & 4 & 13 & 20 & 455 & 5 & 14 & 19 \\
\hline $\begin{array}{l}\text { Sechs Handelsnationen } \\
\text { Ostasiens }\end{array}$ & 860 & 7 & 15 & 24 & 785 & 6 & 12 & 27 \\
\hline
\end{tabular}

Quelle: WTO, International Trade Statistics 2005, Genf, 2005, Tabelle I.3, S. 22.

Anmerkung: Die Differenz zwischen dem Wert der weltweiten Ausfuhren und demjenigen der weltweiten Einfuhren erklärt sich aus der Berücksichtigung der Fracht- und Versicherungskosten (cost of insurance and freight, CIF) im Gesamtbetrag der Importe. 
Der teilweise durch den starken Anstieg der Erdölpreise verursachte Rückgang der Wirtschaftsproduktion wird das Wachstum des Welthandels im Jahr 2005 voraussichtlich bremsen. Die weltweiten Warenausfuhren dürften 2005 mit einer Zunahme um 6,5 Prozent deutlich unter den aussergewöhnlichen Wachstumszahlen des Vorjahres liegen ${ }^{3}$.

In der Rangliste der wichtigsten Ausfuhr- und Einfuhrländer 2004 ist die Schweiz nicht mehr unter den 20 weltweit grössten Exporteuren zu finden (2004 belegte sie den 21. Platz, 2003 war es noch der 19. Rang). Dagegen hält sie 2004, wie bereits 2003, ihren 18. Rang unter den Importeuren. Im Dienstleistungsbereich erreicht die Schweiz Platz 18 unter den Hauptexportländern (2003 war es Platz 16), Platz 27 unter den Importländern. Damit steigt sie in der Rangliste der wichtigsten Akteure des internationalen Handels allmählich ab.

\subsubsection{Internationaler Warenhandel nach Regionen}

Die meisten grossen Regionen verzeichneten 2004 ein robustes Wirtschaftswachstum, welches dem Welthandel einen kräftigen Schub verlieh. Die Zunahme ist vor allem auf das Anziehen der Fertigwarenexporte Nordamerikas (9\%) und der Europäischen Union (7\%) zurückzuführen. Diese beiden Regionen steuern zusammen knapp zwei Drittel des weltweiten Handels mit Fertigwaren bei.

Ein besonderes Merkmal im Jahr 2004 war das ausserordentliche Wirtschaftswachstum in den beiden bevölkerungsreichsten Ländern der Welt, China und Indien $(9,5 \%$ bzw. 7,3\%), sowie die markante Steigerung ihres Handels im zweiten Jahr in Folge. In den Erdöl exportierenden Regionen (Gemeinschaft Unabhängiger Staaten, Mittlerer Osten und Afrika) nahmen die Warenexporte deutlich schneller zu als im weltweiten Durchschnitt.

Die Warenexporte der am wenigsten entwickelten Länder (LDC) nahmen um ein Drittel zu und erreichten im Jahr 200462 Milliarden Dollar. Die Preishausse der Grundprodukte und die Zunahme des Rohölvolumens trugen mit zu diesem positiven Resultat bei. In den nicht Erdöl exportierenden LDC lag das Exportwachstum unter dem Gruppendurchschnitt.

Besonders erwähnenswert war 2004 neben dem Anstieg der Erdölpreise das immer deutlichere Erstarken Chinas als Importeur und Anbieter von Gütern und Dienstleistungen. Das anhaltend dynamische Wachstum der chinesischen Ausund Einfuhren in den letzten Jahren hat China im Jahr 2004 auf den dritten Rang unter den grossen Handelsnationen ansteigen lassen. China entwickelte sich sogar zum ersten globalen Importeur für zahlreiche Basisprodukte und zum weltweit wichtigsten Anbieter einer Reihe von Fertigprodukten. Der rasante Aufstieg Chinas im internationalen Handel erschliesst zahlreichen Ländern neue Gelegenheiten, macht aber auch, besonders im Textilbereich, Anpassungen für andere Länder notwendig.

3 WTO, „International Trade Statistics. Trade Growth in 2005 to slow from record 2004 pace“, Pressemitteilung, Nr. 417, 27. Oktober 2005. 
Der 1. Januar 2005 markierte mit der Aufhebung der Einfuhrquoten im Textilund Bekleidungssektor (nachstehend als "Quoten“ bezeichnet) durch die Vereinigten Staaten, die Europäische Union und Kanada eine weitere Etappe in der Handelsliberalisierung. Die Grosseinkäufer von Bekleidung sind künftig grundsätzlich nicht mehr durch die Quoten gezwungen, ihre Bestellungen in aller Herren Länder - auch in den am wenigsten entwickelten Ländern - aufzugeben. Dieser lange erwartete Schritt hätte den Sieg der Handelsliberalisierung über protektionistische Tendenzen und über die Undurchsichtigkeit des Quotensystems bedeuten sollen. In Wirklichkeit wird das abgekartete Spiel aber weiter gespielt: Mehrere arme Länder, für die Textilien das einzige Eintrittstor zur Industrialisierung bilden, werden zugunsten einiger grosser Staaten, deren Entwicklung bereits in vollem Gange ist, aus dem Rennen geworfen (siehe Rahmentext "Quoten und Textilabkommen“).

\subsubsection{Aussicht eines drohenden Handelskriegs}

Für zahlreiche kleine Produzentenländer des Südens baut sich eine verheerende Schockwelle auf ${ }^{4}$. Bangladesch oder Kambodscha, bei denen Textilien und Bekleidung über drei Viertel der Exporte ausmachen, werden besonders stark in Mitleidenschaft gezogen. Global bahnt sich ein ungleicher Handelskrieg zwischen China, gefolgt von Indien und Pakistan, einerseits und zahlreichen armen Ländern Asiens, Afrikas und Zentralamerikas andererseits an. Arbeitsplatzverluste in Millionenhöhe sind vorprogrammiert; am stärksten wird es die nicht qualifizierten Arbeitnehmerinnen treffen. Die verbleibenden Arbeitsplätze müssen sich gegenüber dem Sozialdumping des Riesens China, der unabhängige Gewerkschaftsorganisationen unterdrückt, behaupten. In diesem Klima haben zum Beispiel die Philippinen oder Bangladesch bereits angekündigt, dass sie ihr Arbeitsrecht lockern würden, während die lokalen Arbeitgeber drohen, Betriebe nach China zu verlagern.

Auch andere, näher bei der Europäischen Union gelegene Länder werden die Auswirkungen der Aufhebung der Quoten zu spüren bekommen, insbesondere Marokko, Tunesien, die Türkei, Bulgarien und Rumänien. In diesen Ländern sind ebenso katastrophale Folgen für die Beschäftigung mit entsprechenden Stabilitäts- und Migrationsproblemen zu befürchten. Diese angrenzenden Länder werden versuchen, die Karte der „höheren Qualitätsklasse“ auszuspielen, was den Wettbewerb mit der europäischen Produktion von Erzeugnissen mit hoher Wertschöpfung verschärfen wird. Genau auf diese Karte setzt die Schweiz, die niemals Importquoten für Textilien eingeführt hat.

4 Natacha David, „La fin des quotas textiles“, Enjeux internationaux, Nr. 7, 1. Quartal 2005. 


\section{Quoten und Textilabkommen}

Das erste Multifaserabkommen (MFA), das 1947 in Kraft getretenen war, führte ein System ein, welches die grossen Märkte des Westens vor den Textil- und Bekleidungsausfuhren aus den Entwicklungsländern schützen sollte. Das Multifaserabkommen wurde dreimal verlängert und führte zum heute beobachteten Zerfall der globalen Produktionskette, indem es die multinationalen Textilkonzerne zwang, ihre Versorgungs- und Fabrikationsquellen zu diversifizieren, anstatt sich bei der Auswahl ihrer Zulieferer konsequent auf die Länder mit den attraktivsten komparativen Vorteilen konzentrieren zu können.

1995 wurde das Multifaserabkommen vom Abkommen über Textilien und Bekleidung (ATC) abgelöst, das im Rahmen der Uruguay-Runde des GATT (1986 bis 1994) ausgehandelt wurde. Das ATC strebte den Abbau der Kontingente innerhalb von zehn Jahren an und sah einen Zeitplan von vier aufeinander folgenden Etappen vor, welche den Liberalisierungsprozess abfedern sollten. Die Importländer haben das Übereinkommen allerdings nur dazu genutzt, Zeit zu gewinnen, indem sie die Kontingente für besonders sensible Produkte erst in allerletzter Minute aufhoben.

Das ATC sieht ausserdem eine befristete Schutzklausel vor, die es einem Land ermöglicht, sich gegen steigende Importe eines Produkts zu schützen, welche der betroffenen inländischen Industrie einen „schwer zu behebenden Schaden“ zufügen, bzw. zuzufügen drohen. Die Schutzklausel wurde von allen Mitgliedern der Welthandelsorganisation (WTO) angenommen. Sie kann selektiv, d.h. je nach Land, in gegenseitigem Einvernehmen oder unilateral angewandt werden, falls nach sechzig Tagen keine Einigung zustande kommt. Die Schutzklausel kann allerdings höchstens während drei Jahren, d.h. spätestens bis 2008, in Kraft bleiben.

Ausserdem ist zu erwähnen, dass auch die Zölle ein Schutzsystem bilden. Die Zölle im Textil- und Bekleidungssektor reichen von 12 Prozent in der Europäischen Union über 15 Prozent in den Vereinigten Staaten bis zu durchschnittlich 29 Prozent in den Entwicklungsländern und liegen damit deutlich über denjenigen in anderen Industriesektoren. Diese Zölle werden vom Abkommen über Textilien und Bekleidung nicht erfasst und bleiben demnach ein potenzielles Instrument, um die Auswirkungen der Quotenabschaffung zu kompensieren. Zahlreiche Entwicklungsländer, welche die chinesische Konkurrenz in Existenznot treibt, hoffen auf "Vorzugszölle", um auf den europäischen oder amerikanischen Märkten Fuss zu fassen.

\subsubsection{Aufsteigende Handelsmacht China}

Seit dem In-Kraft-Treten des Abkommens über Textilien und Bekleidung hat sich der Textilmarkt mit dem WTO-Beitritt Chinas im Jahr 2001 noch stärker angespannt. Gemäss der Weltbank dürfte China, das zum Beitrittszeitpunkt bereits ein knappes Viertel der weltweiten Bekleidungsexporte stellte, bis zum Jahr 2010 die Hälfte davon kontrollieren. Langfristig prognostiziert die Weltbank das Verschwinden der Textilindustrie in der Europäischen Union und in den Vereinigten Staaten. Diese beiden Handelsmächte haben mittlerweile die Schutzklausel ausgelöst, welche die WTO für den Fall einer zu starken Destabilisierung der jeweiligen Märkte vorgesehen hat. Auch Brasilien, Argentinien und Kolumbien haben diesen Mechanismus bereits eingesetzt.

Am 5. September 2005 unterzeichneten die Europäische Union und China ein Abkommen ${ }^{5}$, das die Freigabe von knapp 80 Millionen aus China importierten Kleidungsstücken ermöglichte. Damit wurde die Streitigkeit durch die Aufstockung der für 2005 bewilligten Mengen chinesischer Importe beendet. Dieses

5 International Centre for Trade and Sustainable Development (ICTSD), „China Signs Deal with EU on Impounded Textiles; US Negotiations Stalled“, Bridges. Weekly Trade Digest, Vol. 9, Nr. 29, 7. September 2005 . 
Abkommen ändert den Inhalt des Abkommens vom 10. Juni 2005, welches für zehn Arten chinesischer Textilimporte in die EU die jährliche Zunahme während der drei folgenden Jahre auf maximal 8 bis 12,5 Prozent festlegte. Die fraglichen Bekleidungsartikel, die grösstenteils vor der Verhängung der Einfuhrbeschränkungen bestellt worden waren, wurden nach Ausschöpfung der im Juni 2005 vereinbarten Importquoten in den europäischen Häfen blockiert. Mit dem Abkommen vom September 2005 wurden die Einfuhrkontingente für 2005 erhöht, um die gesperrten Waren freizugeben. China erklärte sich einverstanden, die Hälfte dieser Zunahme von den Importkontingenten für 2006 abzuziehen, während die Europäische Union einwilligte, die restlichen Waren zusätzlich zu den bereits bewilligten Mengen einzuführen. Das neue Arrangement hat zur Folge, dass die bewilligte Menge der chinesischen Textilimporte in die EU im Jahr 2006 niedriger sein wird, als ursprünglich geplant. Die britischen Einzelhändler warnten, dass diese Situation langfristig zu Versorgungsengpässen für chinesische Kleidungsartikel und wahrscheinlich zu höheren Preisen für die europäischen Verbraucher führen werde.

Zwischenzeitlich ist die vierte Verhandlungsrunde zwischen den Vereinigten Staaten und China über ein bilaterales Abkommen, das die Textilstreitigkeit regeln sollte, am 1. September 2005 nach drei Verhandlungstagen gescheitert. Einige Schlüsselfragen sind noch ungelöst (Auslaufen des Abkommens, Anzahl der erfassten Produkte, jährliche Wachstumsrate, usw.). Gleichentags verhängte der US-Ausschuss für die Umsetzung von Textilabkommen ${ }^{6}$ Restriktionen für die Einfuhren von chinesischen Büstenhaltern und Geweben aus synthetischen Filamenten, wobei er den im WTO-Beitrittsprotokoll Chinas verankerten befristeten Schutzmechanismus betätigte ${ }^{7}$. Allerdings verschob der Ausschuss eine Entscheidung über die Einführung von Beschränkungen für vier weitere Bekleidungstypen. Die Vereinigten Staaten hatten die Schutzklausel bereits unter anderen Umständen angewandt, als sie nach dem 1000\%-igen Anstieg der Importe von Hausmänteln, Büstenhaltern und Strickwaren die 2002 aufgehobenen Kontingente wieder einführten.

\subsection{Welthandelsorganisation (WTO)}

Das von den WTO-Mitgliedsstaaten Ende Juli 2004 in Genf unterzeichnete Abkommen sollte der Doha-Runde, die seit dem Scheitern der Ministerkonferenz in Cancún vom September 2003 festgefahren war, neuen Schwung verleihen. In Wirklichkeit drehten sich die Gespräche während des ersten Halbjahrs 2005 im Kreis, so dass der Zeitplan geändert werden musste. Der Sachzwang des amerikanischen Verhandlungsmandats (fast track ${ }^{8}$ ) machte die Ministerkonferenz von Hongkong zu einem entscheidenden Ereignis und führte zu einer Intensivierung der Diskussionen ab Herbst 2005. Der neue Generaldirektor Pascal Lamy, der sein

6 US Committee for the Implementation of Textile Agreements (CITA), für die Verwaltung des Textilhandels der Vereinigten Staaten zuständige Regierungsstelle.

WTO, Protocol on the Accession of China, Artikel 16, 10. November 2001.

8 Beim fast track handelt es sich um das Verhandlungsmandat des amerikanischen Kongresses an die Regierung, das auch den Abschluss von Handelsverhandlungen umfasst. Dieses Mandat läuft Mitte November 2007 aus. Zahlreichen Beobachtern zufolge dürfte es George Bush schwer fallen, ein zunehmend protektionistisch eingestelltes Parlament von einer Verlängerung zu überzeugen. Deshalb ist es für die WTO zwingend wichtig, die Doha-Runde vor diesem Termin abzuschliessen. 
Amt am 1. September 2005 angetreten hatte, scheute keine Mühe, um den Prozess neu zu beleben - allerdings mit geringem Erfolg, so dass die Ambitionen für Hongkong rasch heruntergeschraubt werden mussten.

Jahrbuch 2005, Nr. 1, Kapitel 6, 6.2.3. Ergebnisse des Rahmenabkommens vom Juli 2004, S. 80-84.

\subsubsection{Stand der Verhandlungen im Jahr 2005}

Die Divergenzen darüber, was unter einer „Entwicklungsrunde“ zu verstehen sei und was nicht, erwies sich 2005 als Stolperstein in den Verhandlungen ${ }^{9}$. Nach Ansicht der Industrieländer, einiger Schwellenländer und der Vertreter der Privatwirtschaft sollte die Entwicklungsrunde vor allem auf der Marktliberalisierung und dem daraus resultierenden Wirtschaftswachstum beruhen. Bestimmte Entwicklungsländer und ein Grossteil der Nichtregierungsorganisationen (NGO) dagegen verstehen darunter die Gewährung bzw. Beibehaltung eines echten „Policy Space“, der über die von der WTO eingeräumte besondere und differenzierte Behandlung ${ }^{10}$ hinausgeht (siehe Rahmentext „Policy Space“).

\section{$\square$ Eine Entwicklungsrunde?}

Die im Jahr 2001 in Doha eingeleitete Runde verfolgte das Ziel, die Interessen der Entwicklungsländer in den Mittelpunkt der Verhandlungen zu stellen, die Versprechen, die ihnen während der Uruguay-Runde gemacht worden waren, einzulösen und die sie benachteiligenden Defizite, Ungleichgewichte und strukturellen Ungleichheiten in den bestehenden Abkommen zu beseitigen. Die Entwicklungsländer riefen dieses Ziel kurz vor der Ministerkonferenz von Hongkong zweimal in Erinnerung ${ }^{11}$. In der Erklärung von Doha werden vier Elemente hinsichtlich der Entwicklungsrunde festgehalten: Lösung einer Reihe von Umsetzungsfragen (implementation issues); Beendigung als unfair beurteilter Praktiken (interne Stützung, Exportsubventionen, protektionistische Massnahmen), womit die Länder des Nordens ihre Agrarmärkte schützen, aber gleichzeitig jene des Südens ruinieren; bessere Regelung der besonderen und differenzierten Behandlung, namentlich für die am wenigsten entwickelten Länder; Festigung des Rechts der Mitgliedsstaaten, Zwangslizenzen zu verwenden und aus Gründen der öffentlichen Gesundheit Generika patentgeschützter Medikamente zu importieren.

Indem die Industrieländer den südlichen Ländern eine „Entwicklungsrunde“ „verkauften“, haben sie de facto die Büchse der Pandora geöffnet und eine Grundsatzdebatte über die Gouvernanz der Weltwirtschaft und die Rolle der WTO heraufbeschworen. Dies wirft drei grundlegende Fragen auf:

9 Für Einzelheiten des WTO-Dossiers 2005 siehe ICTSD, Doha Round Briefing Series. Hong Kong Updates, Vol. 3, November 2005.

10 Die WTO-Abkommen enthalten Sonderbestimmungen über die so genannte ,besondere und differenzierte Behandlung“, die den Entwicklungsländern Sonderrechte verleiht. Dazu gehören beispielsweise Bestimmungen, die längere Fristen für die Umsetzung der Abkommen und der Verpflichtungen vorsehen.

11 Die Handelsministerkonferenz der Afrikanischen Union verabschiedete am 24. November 2005 eine Erklärung mit dem Titel „The Arusha Development Benchmarks“. Am 28. November 2005 reichten neun Länder - Argentinien, Brasilien, Indien, Indonesien, Namibia, Pakistan, die Philippinen, Südafrika und Venezuela - dann beim Handels- und Entwicklungsausschuss eine Eingabe mit dem Titel „Reclaiming Development in the WTO Doha Development Round“ ein. 
口 Welcher Zweck soll verfolgt werden : ein Höchstmass an Liberalisierung oder Rahmenbedingungen für die Märkte?

๖ Welcher „Policy Space“ soll den Ländern dabei zugestanden werden?

๑ Welche Entwicklungsziele (Nahrungssicherheit und -souveränität, Wasserversorgung, Erziehung und Basisgesundheit, Umweltschutz usw.) und welche (wirtschaftlichen, sozialen, ökologischen) kollektiven Präferenzen sollen verfolgt werden?

Diese Fragen sprengen den eigentlichen Rahmen der WTO und stehen in einem Spannungsverhältnis zur Schaffung einheitlicher Regeln für alle. Nichtsdestotrotz sollten sie allen Handelsverhandlungen zugrunde liegen. Die Korrelation zwischen Marktliberalisierung, Wirtschaftswachstum und Entwicklung wird von zahlreichen Wirtschaftswissenschaftlern zunehmend in Frage gestellt. In diesem Kontext rief Pascal Lamy kurz vor seinem Amtsantritt als WTO-Generaldirektor zu einem neuen „Konsens“ auf: Die Vorstellung, dass Liberalisierung, Marktöffnung und Privatisierung eine Wunderlösung darstellten, habe ausgedient. Heute könne man nicht mehr davon ausgehen, dass die von der Wirtschaftstheorie prognostizierten Vorteile zwangsläufig und ohne weiteres Zutun in der sozialen Realität Gestalt annehmen. Es sei an der Zeit, den „Konsens von Genf“ zu schmieden; dieser rüttelt nicht an der grundlegenden Idee, wonach alle Beteiligten von der Handelsöffnung profitieren, legt aber Wert auf die Bedingungen, unter denen die theoretischen Gewinne in Kaufkraft, Wachstum und Armutsbekämpfung umgemünzt werden können ${ }^{12}$.

\section{„Policy Space“ \\ Das Konzept „Policy Space” bezeichnet den Handlungsspielraum der Regierungen für die Durchführung von Wirtschaftspolitiken, die auf ihre spezifische Situation und die echten Bedürfnisse ihrer Bevölkerun- gen abgestimmt sind. Die Regierungen nutzen dabei eine breitere Auswahl an Instrumenten und Flexibi- litäten, als von den internationalen Organisationen wie den Bretton-Woods-Institutionen und der WTO zugebilligt wird. Das Konzept wird in der Erklärung der UNCTAD Xla , die vom 13. bis 18. Juni 2004 in São Paulo tagte, festgehalten und damit erstmals in einem zwischenstaatlichen Text anerkannt. Absatz 8 des São Paulo Consensus hält fest, dass es für die Entwicklungsländer in Anbetracht ihrer Entwick- lungsziele besonders wichtig sei, dass alle Länder die Notwendigkeit eines angemessenen Gleichge- wichts zwischen nationalem "Policy Space" und internationalen Disziplinen und Engagements berück- sichtigten („It is particularly important for developing countries, bearing in mind development goals and objectives, that all countries take into account the need for appropriate balance between national policy space and international disciplines and commitments" $)^{b}$.}

Jahrbuch 2005, Nr. 1, Kapitel 6, 6.3.1. 11. Vierjahreskonferenz der UNCTAD, S. 88-92.

Eine Schlüsselfrage hinsichtlich des "Policy Space” lautet, wer die Politiken des Staates nach welchen Interessen zu wessen Vorteil festlegt. Wegen des Defizits an demokratischer Legitimität und/oder Repräsentativität vieler an den WTO-Verhandlungen beteiligter Regierungen genügt es nicht, ihnen diese zentralen Regelungs-, Kontroll- und Umverteilungskompetenzen zurückzugeben, sondern es müssen demokratische Strukturen und Verfahren für die Konsultation und Beschlussfassung in den Staaten selbst und in der WTO geschaffen werden, damit die Bevölkerungen sich Gehör verschaffen können (siehe Punkt 6.5.2).

a UNCTAD, Spirit of São Paulo, doc. TD/L.382, 2004.

b UNCTAD, São Paulo Consensus, doc. TD/410, 2004.

12 Pascal Lamy, zitiert von Thierry Pech und Wojtek Kalinowski, „Politiser la mondialisation. Entretien avec Pascal Lamy“, La Vie des idées, Juni 2005. 
In der Folge wird der Verhandlungsstand 2005 in den wichtigsten Dossiers der WTO - Landwirtschaft, Industriegüter, Dienstleistungen und geistiges Eigentum - im Einzelnen beschrieben.

\section{$\square$ Landwirtschaft}

Der Zugang der Entwicklungsländer zu den Agrarmärkten ${ }^{13}$ der Industrieländer steht im Mittelpunkt der Diskussion. Wie der brasilianische Handelsminister mehrmals betonte, bildet diese Frage den „Motor der Verhandlungen“. Im Landwirtschaftssektor sind die strukturellen Ungleichheiten zwischen Industrie- und Entwicklungsländern am stärksten ausgeprägt. Für die in der G-2014 zusammengeschlossenen Agrarexporteure des Südens würde eine „Entwicklungsrunde", die diesen Namen verdient, beinhalten, dass die reichen Länder Marktöffnungen im Agrarbereich akzeptieren, ohne als Gegenleistung massive Zugeständnisse in anderen Bereichen zu verlangen. Dies stösst bei den Industrieländern auf Widerstand; sie ziehen etwaige Marktöffnungen in der Landwirtschaft nur als Gegenleistung für einen besseren Zugang ihrer Industriegüter und Dienstleistungen zu den Märkten der Entwicklungsländer in Betracht. Der indische Handelsminister übte scharfe Kritik an dieser Haltung und an den „überzogenen“ Forderungen an die Entwicklungsländer; er warf den Industrieländern vor, aus der „Entwicklungsrunde“ von Doha eine „Marktzugangsrunde" gemacht zu haben.

Ab Oktober 2005 war zu beobachten, wie sich die Positionen in Form eines „Blame Game“ (Schwarzer Peter) präziser abzeichneten und ausprägten, wobei jede Partei versuchte, den Einsatz in die Höhe zu treiben, indem sie der Gegenpartei die Schuld für ein etwaiges Verhandlungsfiasko in die Schuhe schob. Die Vereinigten Staaten und die G-20 verlangen von der Europäischen Union und von der G-10 $10^{15}$ mehr Marktöffnung; die G-20 bezeichnete die Vorschläge der Vereinigten Staaten und der Europäischen Union zur Reduzierung der internen Stützung wegen der möglichen Tricks (Verschiebung der Subventionen von einer Kategorie in die andere ${ }^{16}$ ) als enttäuschend, ja irreführend. Die G-20 vertritt zusammen mit der G-33 ${ }^{17}$ und der Gruppe der armen Länder Afrikas, der Karibik und des Pazifik (AKP) die Auffassung, dass die Aspekte der besonderen und differenzierten Behandlung namentlich die Fragen der Nahrungsmittelsicherheit und der ländlichen Entwicklung (besondere Produkte, Schutzmassnahmen) - nicht ausreichend berücksichtigt werden. Die AKP-Länder, die erstmals eine eigene Position

13 WTO, Internetseite für die Landwirtschaft: $<$ www.wto.org/index.htm $>>$ Trade Topics $>$ Goods $>$ Agriculture.

14 Die G-20, die sich für mehr Marktöffnung im Norden einsetzt, umfasst eigentlich 21 Mitglieder: Ägypten, Argentinien, Bolivien, Brasilien, Chile, China, Guatemala, Indien, Indonesien, Kuba, Mexiko, Nigeria, Pakistan, Paraguay, die Philippinen, Simbabwe, Südafrika, Tansania, Thailand, Uruguay und Venezuela. Diese Gruppe vertritt über die Hälfte des Bauernstandes weltweit.

15 Die G-10 unter schweizerischer Leitung umfasst die Industrieländer, welche Nettoimporteure von Agrarprodukten sind und einen starken Schutz für ihre Landwirtschaft fordern: Bulgarien, Island, Israel, Japan, Liechtenstein, Mauritius, Norwegen, die Schweiz, Südkorea und Taiwan.

16 Für die Kategorien der internen Stützung siehe Glossar betreffend die Landwirtschaftsverhandlungen im Jahrbuch 2004, Nr. 1, Kapitel 6, S. 96.

17 Bei der hauptsächlich von Indonesien koordinierten G-33 handelt es sich um eine Allianz von rund vierzig Entwicklungsländern, welche die Prinzipien der Nahrungsmittelsicherheit und des Rechts auf ländliche Entwicklung verteidigen. 
ausgearbeitet hatten ${ }^{18}$, befürchten, dass die Marktöffnungsforderungen der grossen Agrarexporteure sich verheerend auf ihre Landwirtschaft auswirken werden, und verlangen grössere Aufmerksamkeit für das Problem der Präferenzerosion. Die Schweiz unterstützt diese Länder dabei und hat ihre Initiative begrüsst.

Der Spielraum der Verhandlungsteilnehmer ist eng. Der EU-Handelskommissar zog den Zorn der französischen Regierung auf sich, die ihm vorwarf, sein Mandat überschritten zu haben. In der Schweiz bezeichnete das Staatssekretariat für Wirtschaft (seco) unter dem Druck der Agrarlobby ${ }^{19}$ einige Landwirtschaftsvorschläge auf dem Verhandlungstisch der WTO als unannehmbar: Darin wird insbesondere die Absenkung der Zollplafonds sowie die Begrenzung der Anzahl „sensibler Produkte“ verlangt, die zur Förderung der multifunktionellen Rolle der Landwirtschaft besondere Flexibilität geniessen. Der französische Staatspräsident und auch der Bundesrat drohten, ihr „Vetorecht“" auszuüben, falls die Agrarliberalisierungen die Schwelle des Erträglichen und den Rahmen ihrer Agrarpolitik zu stark überschreiten sollten.

\section{$\square$ Industriegüter}

Mit der Zurückziehung der so genannten Singapur-Themen ${ }^{20}$ hat die Liberalisierung des Industriegüterhandels ${ }^{21}$ die Schlüsselposition in den Verhandlungen zurückerobert. Die Vereinigten Staaten, die Europäische Union und die Schweiz richteten weitgehende Forderungen an die Entwicklungsländer:

$\square$ Konsolidierung (Plafonierung) praktisch aller Zölle ;

๖ drastische Senkung der Zölle nach der so genannten ,Schweizer Formel“222.

Diese Massnahmen würden den Entwicklungsländer nicht nur wichtige Haushaltsressourcen, sondern auch einen Grossteil ihres „Policy Space“ entreissen. Die Afrikanische Union, die AKP-Länder und die am wenigsten entwickelten Länder (LDC) bezeichneten die Forderungen als ,gravierende Bedrohung für ihre Entwicklung“. Da die durchschnittlichen Zölle der Entwicklungsländer deutlich über denjenigen der Industrieländer liegen, werde ihnen de facto ein viel grösseres Engagement abverlangt. Dies würde dem Mandat von Doha zuwiderlaufen, das theoretisch auf die speziellen Interessen und Bedürfnisse der Entwicklungsländer ausgerichtet ist und zu ihren Gunsten keine volle Gegenseitigkeit vorsieht.

18 Die AKP-Länder unterbreiteten anlässlich der informellen Sitzung im Rahmen der Sondertagung des Landwirtschaftsausschusses vom 21. Oktober 2005 einen Vorschlag über den Marktzugang, welcher die Schwäche zahlreicher Entwicklungsländer gegenüber der uneingeschränkten Marktöffnung veranschaulichte.

19 Der Schweizerische Bauernverband (SBV) organisierte am 17. November 2005 einen Mobilisierungstag.10'000 Landwirte gingen in Bern auf die Strasse, um ihre Existenzangst öffentlich zu thematisieren.

20 Im Juli 2004 wurden die Themen betreffend Investitionen, Wettbewerb und Transparenz im öffentlichen Beschaffungswesen wegen des Drucks der Entwicklungsländer von der Tagesordnung der WTO gestrichen.

21 WTO, Internetseite für den Marktzugang für Waren: <www.wto.org/index.htm> > Trade Topics > Goods > Market access for goods.

22 Die so genannte „Schweizer Formel“ gilt für jede Produktzeile und bedeutet eine stärkere Absenkung der höheren Tarife sowie den raschen Abbau der Zölle in bestimmten Sektoren wie Fischereiprodukte, Edelsteine und Edelmetalle, usw. 


\section{$\square$ Dienstleistungen}

Im Dienstleistungsbereich ${ }^{23}$ mussten alle WTO-Mitgliedsstaaten ihre Liberalisierungsvorschläge und -begehren bis Ende Juni 2005 einreichen. Die meisten kamen dieser Aufforderung zwar nach, doch halten die Industrieländer die Angebote der Entwicklungsländer für unzureichend. Die Schweiz hatte eine Messmethode ausgearbeitet und der WTO vorgelegt, mit der sich die Angebote nach einer einheitlichen Formel auswerten und vergleichen lassen ${ }^{24}$. Die Europäische Union hatte ein System vorgeschlagen, das von allen Ländern eine Mindestverpflichtung betreffend die Anzahl der abzudeckenden Sektoren und den Liberalisierungsgrad verlangt (Benchmarking). So müssten die Industrieländer neue Verpflichtungen eingehen bzw. ihr Angebot in 139 der 163 von der WTO erfassten Subsektoren verbessern, die Entwicklungsländer in 93 Bereichen.

Die Industrieländer, darunter die Schweiz, verfolgen das Ziel, die Marktöffnung in den Bereichen Finanzdienstleistungen und Versicherungen, Transport, Energie, Telekommunikation und Tourismus für ausländische Dienstleistungserbringer zu ermöglichen. Im Gegenzug sind jedoch die wiederholten Forderungen bestimmter Schwellenländer nach einem besseren Zugang zu den Arbeitsmärkten der reichen Länder (Erbringungsart 4) ${ }^{25}$ immer noch nicht auf positive Resonanz gestossen. Viele Entwicklungsländer haben das System der Mindestverpflichtung abgelehnt, weil es nach ihrer Auffassung den eigentlichen Charakter des Allgemeinen Abkommens über den Dienstleistungshandel (GATS) verändert, welches den einzelnen Ländern grundsätzlich die Wahl lässt, ihrer Situation und ihren Interessen angepasste Liberalisierungsschritte durchzuführen.

\section{$\square$ Geistiges Eigentum}

Um ein positives Signal an die Entwicklungsländer auszusenden, hat die WTO Anfang November 2005 eine Änderung ${ }^{26}$ des Übereinkommens über handelsbezogene Aspekte der Rechte des geistigen Eigentums (TRIPS) ${ }^{27}$ angenommen. Im August 2003, kurz vor der Konferenz von Cancún, hatte die WTO den Entwicklungsländern ohne Produktionskapazitäten die befristete Bewilligung erteilt, Zwangslizenzen zu verwenden und aus Gründen der öffentlichen Gesundheit Generika von patentgeschützten Medikamenten einzuführen. Dieser Beschluss wurde in eine Dauerlösung umgewandelt, die von verschiedenen Bedingungen flankiert ist (Definition, Notifizierung, Beschriftung, usw.), um den freien Verkehr der Generika, die Wiederausfuhr und den Preisdruck zu verhindern.

Die Schweiz, die zusammen mit den USA zu den Ländern gehört, die in dieser Frage die restriktivste Position beziehen, verlautbarte, dass sie mit diesem Konsens leben könne ${ }^{28}$. Viele Organisationen der Zivilgesellschaft kritisierten dagegen

23 WTO, Internetseite für Dienstleistungen : <www.wto.org/indexfr.htm > > Trade Topics $>$ Services.

24 Methodology Developed by Switzerland to Assess Schedules of Commitments, Working Document by Switzerland, Bern, 13. September 2005.

25 Erbringungsart 4 betrifft die zeitlich befristete Anwesenheit natürlicher Personen aus dem Ausland.

26 WTO, „Poorest countries given more time to apply intellectual property rules“, Pressemitteilung Nr. 424, 29. November 2005.

27 WTO, Internetseite für geistiges Eigentum: <www.wto.org/index.htm $>>$ Trade Topics $>$ Intellectual Property.

28 Äusserungen von Luzius Wasescha (seco), zitiert von Ram Etwareea, „L'accord sur les médicaments, de bon augure pour Hongkong“, Le Temps, 7. Dezember 2005. 
die Massnahme und wandten ein, die vorgesehenen Bedingungen und Verfahren seien derart schwerfällig und komplex, dass sie die Umsetzung praktisch verhinderten. In diesem Zusammenhang ist zu erwähnen, dass die im August 2003 angenommene Ausnahmeregelung (Waiver) nicht beansprucht wurde.

Die WTO räumte zudem der Gruppe der am wenigsten entwickelten Länder für die Umsetzung der TRIPS-Bestimmungen eine zusätzliche Frist von siebeneinhalb Jahren - d.h. bis zum 1. Juli 2013 - ein; die betreffenden Länder hatten eine Fristverlängerung von fünfzehn Jahren verlangt.

\subsubsection{Zehnjähriges Bestehen der WTO}

Im Jahr 2005 feierte die WTO ihr zehnjähriges Bestehen ${ }^{29}$. Aus diesem Anlass fanden verschiedene Veranstaltungen statt, die aufzeigten, dass der Stillstand, der die WTO lähmt, nicht nur auf Interessenkonflikte oder Meinungsunterschiede zur „Entwicklungsrunde“ zurückgeht, sondern auch bestimmte tief greifende Umwälzungen widerspiegelt: die neuen geopolitischen Gegebenheiten mit dem Erstarken der Entwicklungsländer, das immer komplexere Wechselspiel der Bündnisse über die Nord-Süd-Kluft hinaus sowie institutionelle Blockaden (siehe oben, Punkt 6.3.1).

\section{$\square$ Aufstieg der Entwicklungsländer}

Eine der wichtigsten Lehren aus dem Jahr 2005 ist, dass die Karten in der Geopolitik des Welthandels zurzeit neu gemischt werden. Das zeigt sich besonders mit dem Aufstieg der Entwicklungsländer, allen voran die aufstrebenden Handelsmächte Brasilien, Indien und Südafrika. Diese Länder verschaffen sich mit zunehmendem Nachdruck Gehör, da sie sich ihres wachsenden Gewichts im Welthandel bewusst sind und die Räderwerke der WTO-Maschinerie immer besser verstehen. Sie haben gelernt, eine geeinte Front zu bilden und sich in verschiedenen strategischen Allianzen - künftig eher auf pragmatischer als auf ideologischer Basis - zu organisieren :

๖ in der G-20 für die Öffnung der Agrarmärkte im Norden ;

口 in der G-33 für Massnahmen im Bereich der Nahrungsmittelsicherheit;

$\square$ in der G-9030, gestern gegen die Singapur-Themen, heute für eine Round for free $e^{31}$.

Bedeutend ist auch die Tatsache, dass diese Blöcke trotz der Unterschiede und der bisweilen divergierenden Interessen der Mitglieder den Druck- und Erpressungsversuchen der Amerikaner und Europäer standgehalten haben, obwohl diese nichts unversucht liessen, um einen Keil zwischen sie zu treiben, besonders indem sie Indien und Brasilien an den Tisch der Entscheidungsträger baten $^{32}$. Die G-20 rückte im September 2005 in Bhurban (Pakistan) noch enger

29 WTO, Internetseite zum zehnjährigen Bestehen der WTO : <www.wto.org/indexfr.htm> > The WTO $>$ WTO $10^{\text {th }}$ Anniversary.

30 Die G-90 vertritt die AKP-Staaten und die am wenigsten entwickelten Länder.

31 Mit Round for free ist eine Verhandlungsrunde gemeint, bei der die Entwicklungsländer keine Konzessionen zu machen haben.

32 Das Rahmenabkommen vom Juli 2004 z.B. wurde von den FIPs (Five Interested Parties) ausgearbeitet, zu denen die Vereinigten Staaten, die Europäische Union, Brasilien, Indien und Australien gehören. 
zusammen und bekräftigte nachdrücklich ihren Willen, die Allianzen mit den anderen Gruppen der Entwicklungsländer zu festigen und dabei deren Anliegen Rechnung zu tragen. Der gemeinsame Nenner ist die Verwirklichung der „Entwicklungsdimension“ der Doha-Runde.

\section{$\square$ Komplexere Interessenlage}

Eine weitere Veränderung besteht in den zunehmend komplexen Verhandlungen und Bündnistaktiken. Die WTO lässt sich nicht mehr auf eine einzige NordSüd-Bruchlinie reduzieren. Die Interessen innerhalb der Blöcke scheren auseinander, die Verhandlungsmacht variiert erheblich. Die WTO ist zu einer mehrpoligen Welt geworden, in der mehrere „Norden“ (Vereinigte Staaten, Europäische Union, G-10) und mehrere „Süden“ (Brasilien als Wortführer des Freihandels, Indien mit eher protektionistischen Tendenzen, afrikanische Länder, die auf die Erhaltung ihres präferenziellen Marktzugangs im Norden bedacht sind) zusammenleben. Diese Verschiedenartigkeit ist im Übrigen mit eine Erklärung, weshalb sich der frühere EU-Handelskommissar Pascal Lamy bei der Berufung an die Spitze der WTO gegen drei Kandidaten des Südens durchsetzte. Die Nord-Süd-Konfrontation, welche die Wahl des vorherigen Amtsinhabers geprägt und zur Aufteilung des Mandats zwischen dem Neuseeländer Mike Moore und dem Thailänder Supachai Panitchpakdi geführt hatte, blieb dieses Mal aus. Als erfahrener Diplomat hat Lamy drei Vertreter der Länder des Südens (Chile, Ruanda, Indien) sowie einen Amerikaner zu stellvertretenden Generaldirektoren ernannt.

\section{$\square$ Festgefahrene Institutionen}

Die dritte Auswirkung der neuen Ordnung betrifft die Institutionen. Die Zeiten, wo einige reiche Länder den Ton angeben konnten, sind vorbei. Nun muss den Entwicklungsländern - 80 Prozent der Mitglieder - Rechnung getragen werden. Sie werden bisweilen in ihrem Kampf von immer besser informierten und reaktionsfähigen NGO unterstützt ${ }^{33}$. Welche Konsultations- und Entscheidungsverfahren sollen eingeführt werden, um repräsentative Verhandlungen zu garantieren, ohne dass eine Blockade entsteht? Wie gelingt es, mit 148 Mitgliedern - in einigen Jahren werden es über 170 sein - unter Berücksichtigung aller Erwartungen und Besonderheiten eine Einigung durch Konsens zu finden? Wie lässt sich die drohende Lähmung des Systems abwenden?

Der WTO-Generaldirektor Pascal Lamy hat sich fest vorgenommen, die Organisation, die er nach dem Scheitern von Cancún als „mittelalterliches Gebilde“ bezeichnet hatte, einer Reform zu unterziehen. Zahlreiche Vorschläge ${ }^{34}$ liegen auf dem Tisch der WTO; sie wurden von einem Ausschuss von acht Mitgliedern unter der Leitung des ehemaligen GATT-Direktors Peter Sutherland ausgearbeitet. Der Katalog der Empfehlungen nennt unter anderem:

33 Zum Beispiel unlängst in den Fällen afrikanische Baumwolle, Singapur-Themen, oder für bestimmte Forderungen hinsichtlich der Nahrungsmittelsicherheit, als die Entwicklungsländer eng mit bestimmten NGO wie Oxfam International, Third World Network, Focus on the Global South, dem International Centre for Trade and Sustainable Development (ICTSD), dem IDEAS Centre usw. zusammenarbeiteten.

34 Consultative Board, The Future of the WTO. Addressing institutional challenges in the new millennium. Report by the Consultative Board to the former Director-General Supachai Panitchpakdi, Genf, 2004. 
๑ Verstärkung des Sekretariats ;

$\square$ Erweiterung der Befugnisse des Generaldirektors;

$\square$ ein Zweistufensystem mit plurilateralen Abkommen, denen nur Mitglieder auf freiwilliger Basis beitreten;

$\square$ Einschränkung des Vetorechts;

๖ Schaffung eines ständigen repräsentativen Rates, usw.

Die gestärkte Machtposition des Sekretariats zeigt sich in der Rolle, die Pascal Lamy bei der Vorbereitung der Ministerkonferenz von Hongkong einnahm. Zum ersten Mal wurde der Entwurf der Ministererklärung vom Generaldirektor selbst verfasst; bislang war dies dem Präsidenten der Konferenz zugefallen.

\subsection{Ministerkonferenz von Hongkong}

Der neue Generaldirektor Pascal Lamy hatte bei seinem Amtsantritt erklärt, auf der Ministerkonferenz von Hongkong sollten zwei Drittel des Wegs hin zum Abschluss der Doha-Runde zurückgelegt werden ${ }^{35}$. Da die Verhandlungen in den wichtigsten Dossiers weitgehend blockiert sind, musste er dieses Ziel rasch nach unten korrigieren. Diese „Auskalibrierung“ ist deshalb besonders wichtig, weil nach dem Fiasko von Cancún ein zweiter Misserfolg, der die WTO institutionell $\mathrm{zu}$ schwächen und die Mitgliedsstaaten $\mathrm{zu}$ entmotivieren drohte, um jeden Preis verhindert werden musste. So wurde beschlossen, dass die Konferenz vor allem dazu dienen sollte, sich aus bestimmten Sackgassen herauszumanövrieren, in spezifischen Punkten Fortschritte zu erzielen und einen klaren Marschplan für den weiteren Prozess festzulegen.

\subsubsection{Position der Schweiz}

In dieser Situation beschloss der Bundesrat, das für die Konferenz von Cancún angenommene Verhandlungsmandat nicht zu verändern, behielt sich aber die Möglichkeit vor, bei Bedarf während der Ministerkonferenz eine telefonische Konsultation mit dem Bundesratskollegium durchzuführen. Die materielle Position wurde nicht geändert: ehrgeizige Liberalisierung für Dienstleistungen und Industriegüter, begrenzte Öffnungen im Agrarbereich unter Wahrung der nicht handelsbezogenen Anliegen und der Vereinbarung der Interessen der Exportund Importländer ${ }^{36}$. Ausserdem befürwortet der Bundesrat verbesserte Handelsregeln und die Einteilung der Entwicklungsländer in neue Kategorien. Bundesrat Joseph Deiss, der die schweizerischen Delegation leitete, erklärte in seiner Ansprache vor der Plenarsitzung zur Eröffnung der Ministerkonferenz in Hongkong, ein Scheitern der Verhandlungen liege in Niemandes Interesse. Die Geschichte habe gezeigt, dass die Handelsöffnung das Wachstum fördere. Heute

35 Pascal Lamy gab dieses Ziel am 14. September 2005 dem Handelsverhandlungsausschuss und gleichentags den Medien anlässlich seines ersten Briefings bekannt.

36 Diese Formulierung wurde von Bundesrat Joseph Deiss in seiner Ansprache auf der Plenarversammlung der WTO-Ministerkonferenz in Hongkong aufgegriffen. WTO, Déclaration de S.E. M. Joseph Deiss, conseiller fédéral, chef du Département fédéral de l'économie, doc. WT/MIN(05)/ST/2, 15. Dezember 2005. 
müsse man darüber nachdenken, mit welchen Mitteln dafür gesorgt werden kann, dass diese Vorteile möglichst Vielen zugute kommen ${ }^{37}$.

Anfang Dezember warf economiesuisse der Regierung vor, ihre Position sei von einer „unzeitgemässen und überspitzten agrarprotektionistischen Haltung“ gekennzeichnet, welche den wirtschaftlichen Realitäten und Prioritäten der Schweiz nicht gerecht werde ${ }^{38}$. Der Arbeitgeberverband appellierte ausserdem an den Bundesrat, keine „Bremserrolle“ zu spielen und die ,gewichtigen Offensivinteressen der Wirtschaft" auf den Dienstleistungs- und Industriegütermärkten $\mathrm{zu}$ verteidigen.

Die Schweizer WTO-Koordination ${ }^{39}$, zu welcher Gewerkschaften, Bauernverbände sowie entwicklungspolitische und umweltpolitische NGO gehören, forderte den Bundesrat auf, sich ,für eine Neuausrichtung der laufenden Verhandlungsrunde einzusetzen“, die zu stark auf den Marktzutritt konzentriert sei. Dazu „müsse die Schweiz ihre übertriebenen Liberalisierungsforderungen bei den Industrie- und Dienstleistungsmärkten aufgeben, weil diese den armen Ländern mehr Schaden als Nutzen brächten" ${ }^{40}$.

\section{$\square$ Hauptergebnisse}

Die Ministerkonferenz in Hongkong war von oft zähen und angespannten Diskussionen geprägt. Die Vereinigten Staaten und die G-20 setzten die Europäische Union massiv unter Druck und beschuldigten sie, mit ihrer Weigerung gegen weitere Agrarkonzessionen die Verhandlungen lahmzulegen. In geopolitischer Hinsicht waren neue Solidaritätsbekundungen unter den Ländern des Südens zu beobachten. Die G-20, die G-33, die AKP-Länder und die am wenigsten entwickelten Länder organisierten erstmals in der Geschichte der WTO zusammen eine Pressekonferenz und trugen eine gemeinsame Position vor. Allerdings ist diese Allianz zu relativieren, da in den verschiedenen informellen Verhandlungssitzungen (green rooms ${ }^{41}$ ), die im Laufe der Woche stattfanden, auch Interessenunterschiede zwischen den Entwicklungsländern auftraten.

Da kein Mitgliedsstaat der WTO die Verantwortung für einen Misserfolg auf sich nehmen wollte, mündete die Ministerkonferenz in Hongkong in einer Ministererklärung42. Darin wurden im Grossen und Ganzen die wichtigsten Punkte der Einigung vom Juli 2004 bekräftigt, die als Verhandlungsrahmen gedient hatte. Die für die Fortsetzung der Verhandlungen entscheidenden Hauptpunkte sind folgende:

๑ Landwirtschaft: Trotz des Widerstands der Europäischen Union und der G-10 wurde das Jahr 2013 als Termin für den Abbau aller Formen von

37 Ibid.

38 economiesuisse, ,Welthandelsorganisation (WTO). Vor der Ministerkonferenz in Hongkong“, Dossierpolitik, 29. November 2005; „Schweizer Wirtschaft für offensives Vorgehen an WTO-Ministerkonferenz in Hongkong“, Medienmitteilung, 30. November 2005.

39 Die Mitglieder des Komitees der Schweizer WTO-Koordination sind: Alliance Sud, Erklärung von Bern, Pro Natura, Schweizerischer Bauernverband, Schweizerischer Gewerbebund, Uniterre.

40 Schweizer WTO-Koordination, „Schweizer WTO-Koordination fordert echte Entwicklungsrunde“, Pressemitteilung, 5. Dezember 2005.

41 „Informelle“ Verhandlungssitzungen mit einer begrenzten Anzahl (generell rund 30) Länder, welche für die verschiedenen einander gegenüberstehenden Interessen und Positionen mehr oder weniger repräsentativ sind.

42 WTO, Ministerial Declaration vom 18. Dezember 2005, doc. WT/MIN(05)/DEC, 2005. 
Exportsubventionen festgelegt. Das Bedürfnis der Entwicklungsländer, einige für die Nahrungsmittelsicherheit wichtige Spezialprodukte von den Zollreduktionen auszunehmen, wurde bestätigt;

- Baumwolle: In der Baumwollthematik, die erneut Schlagzeilen machte, wurden nur dürftige Fortschritte verzeichnet. Die Vereinigten Staaten zeigten sich zwar aufgeschlossener für die Forderungen der betroffenen afrikanischen Länder (Benin, Burkina Faso, Mali und Tschad), blieben aber im Wesentlichen unnachgiebig. Die Frage soll im Rahmen des Landwirtschaftsabkommens behandelt werden. Die Subventionen der Industrieländer für Baumwollexporte müssen bis Ende 2006 abgebaut werden. Für die interne Stützung hingegen - die Hauptursache für Handelsverzerrungen und Dumping, das die Weltmarktpreise unter Druck setzt - wurde nichts beschlossen;

ـ Industriegüter: Für die Zollsenkungen wurde das Prinzip der so genannten „Schweizer Formel“ mit je nach Entwicklungsstand der Länder unterschiedlichen Koeffizienten angenommen und die Lancierung von Initiativen für eine verstärkte und rasche Liberalisierung in bestimmten Sektoren beschlossen;

- Dienstleistungen: Ein Verhandlungsrahmen ${ }^{43}$ wurde trotz der deutlichen Ablehnung seitens der G-90 angenommen, welche kritisierte, dass der Inhalt des Verhandlungsrahmens die Industrieländer zu stark bevorzuge, und dass der Rahmen selbst auf untransparente und undemokratische Weise definiert worden sei. De facto ermöglicht der Kompromiss verstärkten Druck für weiter gehende und zwingendere Verpflichtungen im Bereich der Marktöffnung sowie die Aufnahme plurilateraler Verhandlungen ${ }^{44}$;

$\square$ Besondere und differenzierte Behandlung: Nur ein einziger der fünf Diskussionsvorschläge der am wenigsten entwickelten Länder führte zu vertieften Gesprächen und konkreten Vorschlägen. Die Entwicklungs- und Transitionsländer müssen bis 2008 für Produkte aus den am wenigsten entwickelten Ländern einen zoll- und kontingentfreien Marktzugang anbieten. Jedoch können sie bis zu drei Prozent der Produkte von diesen Präferenzen ausnehmen. Mit anderen Worten könnten beispielsweise die Vereinigten Staaten ihre Textilindustrie weiterhin gegen die Einfuhren aus Bangladesch schützen. Dieses „Entwicklungspaket“ enthält ausserdem Zusagen für handelsbezogene technische und finanzielle Hilfsprogramme.

Die Mitgliedstaaten änderten den Zeitplan für die Verhandlungen, um die DohaRunde bis Ende 2006 unter Dach und Fach zu bringen. Der 30. April 2006 und der 31. Juli 2006 wurden als Stichtage für die Festlegung der Modalitäten bzw. für die ersten konkreten Vorschläge bestimmt. Die Möglichkeit einer weiteren Ministerkonferenz (im Frühjahr in Genf) wurde angesprochen, aber nicht bestätigt.

43 WTO, Ministerial Declaration, op. cit., Anhang C.

44 Im WTO-Jargon bezieht sich „Plurilateralismus“ auf Verpflichtungen, die nur einige Mitglieder mit dem Ziel eingehen, bestimmte Themen um einen harten Kern von Ländern herum voranzutreiben und abzuwarten, dass die übrigen Länder auf den fahrenden Zug aufspringen. 


\section{$\square$ Reaktionen}

Bundesrat Joseph Deiss begrüsste anlässlich einer Pressekonferenz in Hongkong den Erfolg der Veranstaltung ${ }^{45}$. Nach seiner Auffassung stellt die Ministererklärung eine ausgezeichnete Grundlage für die weitere Arbeit im Rahmen der Doha-Runde dar. Die Beschlüssen nähmen die in Genf geleisteten Fortschritte nicht nur zur Kenntnis, sondern enthielten zahlreiche Ergänzungen, bisweilen auch zu heiklen Fragen. Bundesrat Deiss begrüsste mehrere Durchbrüche, besonders bei der Behandlung der für die Entwicklungsländer sensiblen Agrarprodukte und beim Abbau der Exportbeihilfen bis 2013. Die Texte über die Industriegüter und die Dienstleistungen bezeichnete er als zufriedenstellend, auch wenn das Niveau der Ambitionen noch präzisiert werden müsse. Dagegen bedauerte er, dass dem erweiterten Schutz für geografische Herkunftsangaben nur geringe Bedeutung beigemessen wurde, und erklärte, er hätte sich mehr Grosszügigkeit für das „Entwicklungspaket“ gewünscht, das sämtliche Produkte der am wenigsten entwickelten Länder hätte erfassen sollen.

economiesuisse bedauerte den „minimalen Fortschritt“ der Konferenz gerade ,in den für die Schweizer Wirtschaft entscheidenden Bereichen wie Marktzugang für Industriegüter, Liberalisierung des Dienstleistungshandels und Handelserleichterungen" ${ }^{646}$.

Die in Hongkong vertretenen schweizerischen NGO (Alliance Sud, Erklärung von Bern, Pro Natura) erklärten sich ,enttäuscht und beunruhigt“ über die Ergebnisse der Ministerkonferenz, welche „nur wenige Elemente einer wirklichen Entwicklungsrunde enthält" ${ }^{“ 47}$. Der Termin für die Streichung der Exportbeihilfen wurde von 2010 auf 2013 verschoben; das „Entwicklungspaket“ sei ein „Ablenkungsmanöver"48, die Probleme der afrikanischen Baumwollproduzenten blieben ungelöst, die „Industrieländer“ - darunter die Schweiz - „fordern von den Entwicklungsländern einen weitreichenden Marktzugang im Dienstleistungsbereich und bei den Industriegütern“"

\subsection{Tätigkeiten der entwicklungspolitischen NGO}

Zur Arbeit der entwicklungspolitischen Nichtregierungsorganisationen im Bereich der internationalen Handelsbeziehungen sind für 2005 Neuigkeiten zu vermelden. Drei Ereignisse verdienen besondere Erwähnung: die weltweite Aktionswoche, die breit angelegte Konsultation von Alliance $\mathrm{Sud}^{49}$ bei den südlichen NGO sowie das Seminar der Erklärung von Bern über die Finanzdienstleistungen.

45 Statement of Federal Councilor Joseph Deiss, Head of Delegation Meeting, Hongkong, 18. Dezember 2005 (schweizerische Stellungnahme nach der Abschlusssitzung der Delegationsleiter).

46 economiesuisse, „WTO: Nur minimaler Fortschritt in Hongkong“, Pressemitteilung, 19. Dezember 2005.

47 Alliance Sud/Erklärung von Bern/Pro Natura, „Schweizer NGOs enttäuscht und beunruhigt“, Medienmitteilung, 18. Dezember 2005.

48 Alliance Sud/Erklärung von Bern/Pro Natura, „Das Entwicklungspaket ist ein Ablenkungsmanöver“, Pressemitteilung, 16. Dezember 2005.

49 Alliance Sud ist seit August 2005 der neue Name der Arbeitsgemeinschaft der Hilfswerke Swissaid/Fastenopfer/Brot für alle/Helvetas/Caritas/HEKS. 


\subsubsection{Aktionswoche über den Handel}

Hunderte von NGO, Basisbewegungen und Netze mobilisierten in achtzig Ländern weltweit über 10 Millionen Menschen für die Teilnahme an der Globalen Aktionswoche über Handel ${ }^{50}$. Die Veranstaltung fand vom 10. bis 16. April 2005 unter dem Slogan „Trade justice not free trade“ statt. Während einer Woche sollte eine breit angelegte internationale Kampagne durchgeführt werden, die aus dezentralisierten Veranstaltungen mit jeweils eigenem Inhalt und Form bestand. Dabei wurden drei Ziele verfolgt: den Mythos der Marktliberalisierung und der Privatisierung der öffentlichen Dienste als Wunderlösung für die Entwicklung - gemäss der Doktrin der G-8, des IWF, der Weltbank, der WTO und zahlreicher Regierungen - zu widerlegen; auf multilateraler Ebene und mit bilateralen und regionalen Handelsabkommen Alternativen für das von der WTO erstellte Handelssystem zu fördern; von der Handelspolitik zu verlangen, dass sie den Menschenrechten, der Armutsverringerung, der Gesundheit und Erziehung Priorität einräumt und dazu insbesondere die multinationalen Konzerne verbindlichen Normen unterstellt.

In der Schweiz beteiligten sich mehr als fünfzig Nichtregierungsorganisationen an zahlreichen über das ganze Land verstreuten Veranstaltungen. In der Westschweiz wurde der Schwerpunkt auf die Kampagne "GATS und Demokratie“ gelegt, die im September 2003 von der Alliance Sud ${ }^{51}$ bei lokalen Parlamentariern lanciert und ab 2004 von Attac weitergeführt wurde ${ }^{52}$. Nach Auffassung der Schweizer NGO verfolgt der Bundesrat im Rahmen des Allgemeinen Abkommens über den Handel mit Dienstleistungen (GATS) eine „offensive“ Liberalisierungsstrategie. Die Kampagne versuchte deutlich zu machen, dass die kantonalen und lokalen Behörden dem Bundesrat „fast blind vertrauen“ und „weitgehend Gefolgschaft leisten“, und dass sie Schwierigkeiten haben, die mittel- und langfristigen Auswirkungen des Abkommens abzuschätzen. Alliance Süd und Attac beurteilen den Mangel an Transparenz und das Demokratiedefizit, welche die Verhandlungen über die Dienstleistungen charakterisieren, als inakzeptabel. Einige Parlamentarier wurden durch die Kampagne sensibilisiert und setzten eine Diskussion in Gang. Das Ergebnis war, dass sich über siebzig Gemeinden in der Schweiz - wie gut tausend Gemeinden in Europa - zu „GATS-freien Zonen“ erklärten.

\subsubsection{Konsultation bei den NGO des Südens über den Welthandel}

Anlässlich des zehnjährigen Bestehens der WTO organisierte Alliance Sud eine umfassende Konsultation der Nichtregierungsorganisationen im Süden. 57 NGO das sind knapp die Hälfte der befragten Organisationen, grösstenteils Partner der Alliance-Sud-Hilfswerke - beantworteten den Fragebogen, und rund fünfzehn Delegierte aus Afrika, Lateinamerika und Asien beteiligten sich am Symposium vom 1. und 2. Juni 2005 in Bern zum Thema „Welcher Handel nützt den Armen?“.

50 Globale Aktionswoche über Handel: <www.april2005.org>.

51 Im Namen der Schweizer WTO-Koordination, <www.alliancesud.ch > > Themen/Kampagnen $>$ Handel > Archiv > Kampagne GATS und Demokratie.

52 Für die Einzelheiten der Kampagne und die Liste der Gemeinden wird auf die Website <www.suisse. attac.org $>$ verwiesen. 
Vier Ziele standen dabei im Vordergrund: das Verhältnis zwischen Handel und Entwicklung, die Rollen und Kompetenzen der WTO, die möglichen Alternativen sowie den Inhalt einer echten „Entwicklungsrunde“ neu zu überdenken; die Reflexion auf diese Fragen auszudehnen, ohne sich von vornherein von den Grenzen eines von der Sichtweise der WTO geprägten Entwicklungsmodells einengen zu lassen; das Problem, von den Anliegen der betroffenen Bevölkerungen ausgehend, bei der Wurzel anzupacken; die Verbindungen zwischen Lobby-NGO des Nordens und Basisorganisationen im Süden zu dynamisieren.

\section{Reaktionen auf die Handelsliberalisierung}

Aus den Konsultationen mit der Zivilgesellschaft der Entwicklungsländer liessen sich vier miteinander verbundene Punkte herauskristallisieren.

1. Den Handel in nationale Entwicklungsstrategien integrieren bzw. wieder integrieren: Nationale Strategien dürfen nicht von aussen aufgezwungen werden, sondern sie müssen je nach den Besonderheiten des jeweiligen Landes und den Bedürfnissen der Bevölkerungen endogen erarbeitet werden. Es geht um die Notwendigkeit und das Recht der Staaten des Südens und des Nordens, einen echten „politischen Handlungsspielraum" zu behalten, neu zu schaffen oder zurückzuerobern.

2. Die Architektur des internationalen Handels von der lokalen und regionalen Ebene ausgehend überdenken: Der Welthandel stellt eine unumgängliche Realität dar - kein Land kann autark leben oder sich der Globalisierung entziehen - und ist per se weder gut noch schlecht. Die Wirtschaftspolitik muss prioritär die Entwicklung eines nationalen Marktes und die regionale Integration verfolgen, bevor die Beteiligung am Weltmarkt und die Exportförderung um jeden Preis angestrebt wird.

3. Die WTO soll reformiert werden: Die überwiegende Mehrheit der Teilnehmer betonte die Notwendigkeit einer multilateralen Regelungsinstanz für den Welthandel. Im Zusammenhang mit den Reformvorschlägen wurden anlässlich des Forums besonders drei Fragen erörtert: bessere Ausschöpfung des bestehenden Handlungsspielraums und der Flexibilitäten in der WTO; Schaffung von transparenteren und demokratischeren Strukturen und Verfahren; strukturelle Unterscheidung der Länder mit stärkerer Berücksichtigung des jeweiligen Entwicklungsstands. Diese notwendige Differenzierung darf jedoch nicht mit der Einführung neuer Kategorien von Entwicklungsländern in der WTO verwechselt werden.

4. Alternative Wirtschafts- und Handelsmodelle entwickeln und fördern: Solche Modelle müssen glaubwürdige Alternativen zum vorherrschenden System bieten. Drei Denkansätze wurden erwähnt: Eine „Alternative“ bedeutet nicht einen Rückzug aus den internationalen Märkten, sondern eine Wiedereinbettung der Wirtschaft in die Gesellschaft. Die „Essenz" der Entwicklung besteht darin, dass die Vielfalt der Wahlmöglichkeiten vergrössert und die Freiheit der Einzelnen, der Gemeinschaften, Gesellschaften und Nationen, zu wählen, was für sie am besten ist, geachtet wird. Ausserdem soll beim fairen Handel die Kohärenz zwischen lokaler und regionaler Ebene gewährleistet sein.

Siehe Michel Egger, Für mehr Vielfalt in der Handelspolitik, Dossiers Global+ (Alliance Sud), Nr. 5, 2005. Das Dossier fasst die Ergebnisse des Symposiums zusammen und enthält als Ergänzung die verschiedenen Beiträge in der Originalsprache.

Die Beiträge ${ }^{53}$ der NGO des Südens spiegeln neben den reichen Erfahrungen auch sehr vielseitige Standpunkte wider. Bei der Bestandsaufnahme sind sich jedoch alle befragten Organisationen in einem Punkt einig: Das ,neoliberale Credo“, wonach die Handelsliberalisierung und die Integration in den Weltmarkt den Schlüssel für die Entwicklung und die Armutsbekämpfung bilden, ist eine „Mystifizierung“, die keineswegs der erlebten Realität der Bevölkerungen entspricht. Gemäss diesen NGO haben zehn Jahre WTO und Programme des

53 Michel Egger, Welcher Handel für die Armen?, Dossiers Global+ (Alliance Sud), Nr. 4, 2005. Das Dossier enthält eine Zusammenstellung der 57 Beiträge der Partner aus dem Süden. 
Internationalen Währungsfonds (IWF) im Gegenteil vor allem dazu beigetragen, den Handlungsspielraum der Staaten zu untergraben, die Machtstellung der multinationalen Konzerne zu festigen, die öffentlichen Dienste zu privatisieren, die Ungleichheiten zu verschärfen und die Umwelt zu zerstören.

Das Problem ist de facto nicht der Welthandel an sich, sondern die Art und Weise, wie die Handelsbeziehungen und -regeln festgelegt werden. Diese Beziehungen und Regeln widerspiegeln und stärken die „,bestehenden Asymmetrien“. Zwar ist die Rede von „Freihandel“ für alle, aber nur eine Minderheit - die Länder des Nordens, die multinationalen Konzerne und die Eliten der Entwicklungsländer - ziehen Vorteile daraus. So werden nicht nur völlig ungleiche Akteure zueinander in Konkurrenz gestellt, sondern den Stärkeren wird überdies erlaubt, ihre sensiblen Sektoren zu schützen und unlautere Praktiken fortzusetzen.

\subsubsection{Seminar über die Dienstleistungen}

Die Erklärung von Bern organisierte am 6. Dezember 2005 in Bern eine internationale Tagung zum Thema „Freier Marktzutritt für Banken: Wer bezahlt den Preis ?"54. Ziel des Seminars war es, ein bisher wenig diskutiertes Kapitel der WTO-Verhandlungen - die Liberalisierung der Finanzmärkte - zu beleuchten. Dieses Thema war in der WTO von Anfang an sehr umstritten. Zahlreiche Entwicklungsländer und vor allem Schwellenländer lehnten ein Abkommen lange Zeit ab und wiesen auf die strukturellen Ungleichheiten zwischen den Ländern des Nordens und des Südens hin. Trotzdem wurde das Finanzdienstleistungsabkommen Ende 1997 - im Jahr der Finanzkrise in Asien - angenommen und trat 1999 in Kraft.

Der Finanz- und Bankensektor zählt zu den prioritären Interessen der Schweiz. Ohne ins Detail zu gehen, hat die Schweiz - wie auch die Europäische Union und die Vereinigten Staaten - weitgehende Marktöffnungsbegehren an die Länder des Südens gestellt. Diese Länder sind überzeugt, dass ein liberalisierter Finanzmarkt das Wirtschaftswachstum ankurbeln und den Lebensstandard aller WTO-Mitglieder anheben wird und somit ein Schlüsselelement der DohaRunde bildet. Diese These sowie die wichtigsten diesbezüglichen Argumente Innovation, Technologietransfer, sinkende Kosten der Finanzdienstleistungen, Verbesserung der Wettbewerbsfähigkeit der lokalen Gesellschaften - wurden anlässlich des Symposiums untersucht und zur Diskussion gestellt. Das Symposium befasste sich eingehend mit der Politik der ausländischen Grossbanken in den Entwicklungsländern, genauer mit den Konsequenzen für die lokale Bankwirtschaft, für Kredite an Kleinunternehmen und an die Bevölkerung in ländlichen Gebieten sowie mit dem Beitrag an die Doha-Entwicklungsrunde.

54 Erklärung von Bern, Internetseite der Tagung „Freier Marktzutritt für Banken: wer bezahlt den Preis ?“, Tagungsprogramm und Text der wichtigsten Referate : <www.evb.ch/de/p10318.html>. 


\section{QUELLEN}

\section{Welthandelsorganisation (WTO)/World Trade Organization (WTO)}

Consultative Board, The Future of the WTO. Addressing institutional challenges in the new millennium. Report by the Consultative Board to the former Director-General Supachai Panitchpakdi, Genf, 2004.

Déclaration de S.E. M Joseph Deiss, conseiller fédéral, chef du Département fédéral de l'économie, doc. WT/MIN(05)/ST/2, 15. Dezember 2005.

International Trade Statistics 2005, Genf, 2005.

International Trade Statistics, „Trade Growth in 2005 to slow from record 2004 pace“, Pressemitteilung, Nr. 417, 27. Oktober 2005.

Ministererklärung vom 18. Dezember 2005, doc. WT/MIN(05)/DEC, Genf, 2005.

„Poorest countries given more time to apply intellectual property rules“, Pressemitteilung Nr. 424, 29. November 2005.

Protocol on the Accession of China, 10. November 2001.

World Trade Report 2005, Genf, 2005.

\section{Nichtregierungsorganisationen und Lobby-Gruppen}

Alliance Sud/Erklärung von Bern/Pro Natura, „Das Entwicklungspaket ist ein Ablenkungsmanöver“, Pressemitteilung, 16. Dezember 2005.

Alliance Sud/Erklärung von Bern/Pro Natura, ,Schweizer NGOs enttäuscht und beunruhigt“, Medienmitteilung, 18. Dezember 2005.

economiesuisse, „Schweizer Wirtschaft für offensives Vorgehen an WTO-Ministerkonferenz in Hongkong", Medienmitteilung, 30. November 2005.

economiesuisse, „WTO: Nur minimaler Fortschritt in Hongkong“, Medienmitteilung, 19. Dezember 2005.

Egger M., Für mehr Vielfalt in der Handelspolitik, Dossiers Global+ (Alliance Sud), Nr. 5, 2005.

Egger M., Welcher Handel für die Armen?, Dossiers Global+ (Alliance Sud), Nr. 4, 2005.

Erklärung von Bern, Internetseite der Tagung „Freier Marktzutritt für Banken: wer bezahlt den Preis ?“” $<$ www.evb.ch/d/p10318.html>.

Schweizer WTO-Koordination, „Schweizer WTO-Koordination fordert echte Entwicklungsrunde“, Pressemitteilung, 5. Dezember 2005.

\section{INTERNET-ADRESSEN}

Afrikanische Union: <www.uneca.org >.

Agence de coopération et d'information pour le commerce international (ACICI) : <www.acici.org $>$.

Alliance Sud: <www.alliancesud.ch>.

Erklärung von Bern (EvB) : <www.evb.ch>.

Europäische Union (EU) : <www.europa.eu.int>.

Globale Aktionswoche für Handel: <www.april2005.org >.

IDEAS Centre: <www.ideascentre.ch>.

International Centre for Trade and Sustainable Development (ICTSD) : $<$ www.ictsd.org $>$.

Kampagne GATS und Demokratie: $<w w w . a l l i a n c e s u d . c h>$ und $<w w w . s u i s s e . a t t a c . o r g>$.

Office of the United States Trade Representative: <www.ustr.gov>.

Oxfam International : <www.oxfaminternational.org $>$.

Schweizerischer Bauernverband (SBV): $<w w w$.bauernverband.ch $>$.

Staatssekretariat für Wirtschaft (seco) : <www.seco-admin.ch $>$.

Third World Network: $<$ www.twnside.org.sg $>$.

UNCTAD : <www.unctad.org $>$.

UNO: <www.un.org>.

Verband der Schweizer Unternehmer (economiesuisse) : <www.economiesuisse.ch $>$.

Weltbank: <www.worldbank.org>.

Welthandelsorganisation (WTO) : <www.wto.org >

Welthandelsorganisation (WTO), Zehnter Jahrestag: <www.wto.org/english/thewto_e/thewto_e.htm>. 